9

\title{
How embryonic hearts pump: insights from multi-scale microscopy in zebrafish
}

Anjalie Schlaeppi $^{1,2}{ }^{2}$, Madison Hummel ${ }^{1}$, Michaela Mickoleit ${ }^{2}$, Nicola Gritti $^{1,3}$, Kyle Harrington 4,5 , Rory Power ${ }^{1,6}$, Jan Huisken ${ }^{1,2}$ $1_{\text {Morgridge Institute for Research, USA. }}{ }^{2}$ Max Planck Institute of Molecular Cell Biology and Genetics, Germany. ${ }^{3}$ EMBL, Spain. ${ }^{4}$ Max Delbrueck Center for Molecular Medicine, Germany. ${ }^{5}$ University of Idaho, USA. ${ }^{6}$ EMBL, Germany

\section{Abstract Text}

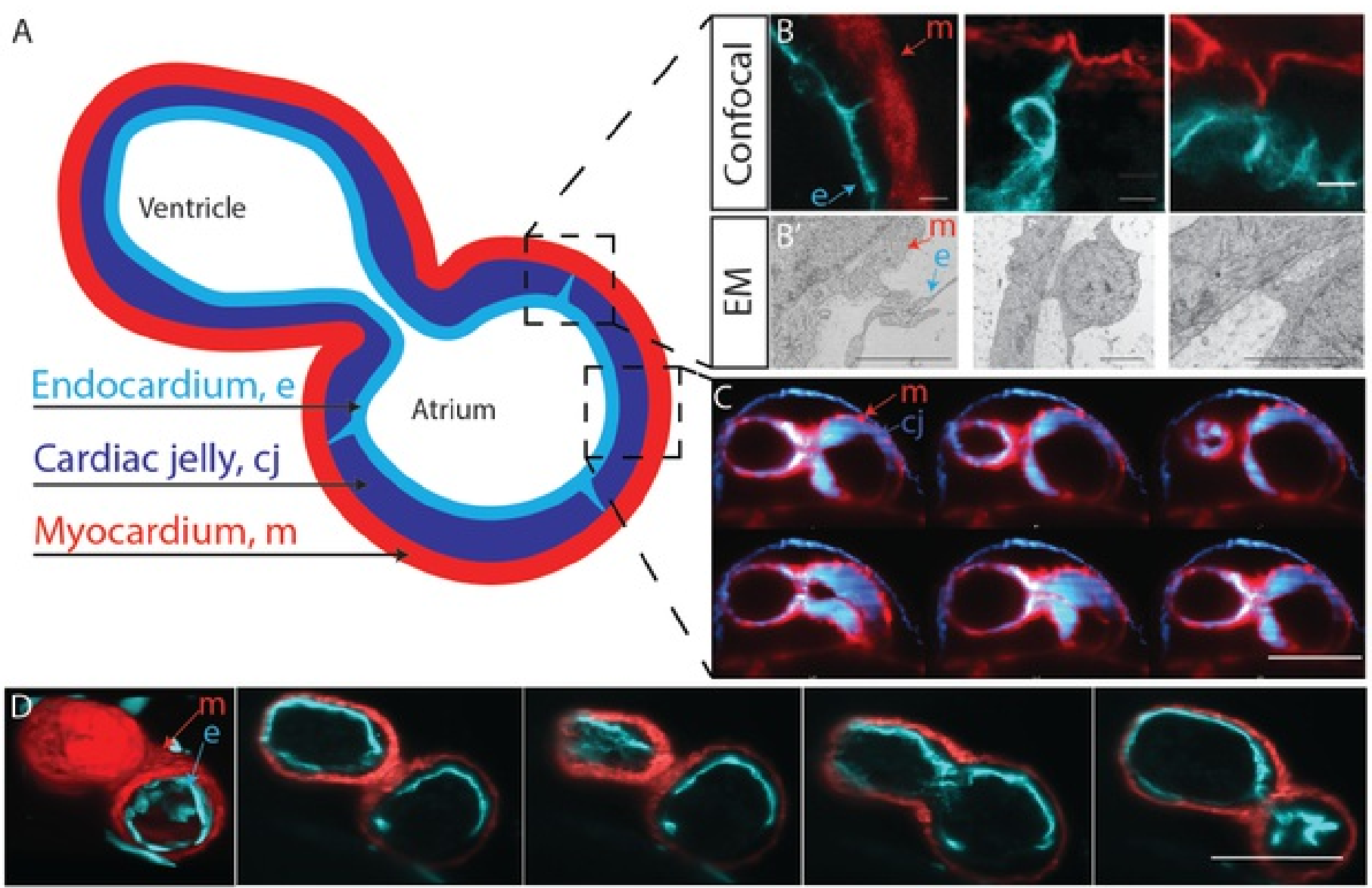

Details of early heart morphogenesis and function still remain unclear. Because the heart undergoes major remodeling during its development, the complex mechanical and the resulting 
physiological properties change while the heart matures. The early embryonic heart is valve-less and made of three layers: the myocardium, the muscle cell layer generating force; the cardiac jelly, an extracellular matrix present only in the embryonic heart; and the endocardium, a single cell layer which underlines the cardiac jelly and encloses blood flow. A complete picture of the interplay and the mechanics of these cardiac constituents is still lacking as the dynamic and molecular regulation of heart development can only be studied in the live, beating embryonic heart.

The zebrafish Danio rerio has become an important cardiac research model as its peripheral heart location and translucency during early development make it ideal for imaging studies. However, resolving moving objects, such as in vivo beating hearts, is technically challenging. Our lab has developed state-of-the-art light sheet (or selective plane illumination microscopy, SPIM) [1] instruments and protocols tailored to live zebrafish imaging, with a particular focus on sample health. This, combined with the speed of light sheet allows us to follow development over days. Recently, we have imaged and reconstructed beating zebrafish hearts in vivo in 3D across time with a dedicated instrument and post-acquisition synchronization of movie stacks [2].

Here we present some of our latest findings on how the early heart pumps blood in the absence of valves. With our cardiac SPIM and multimodal imaging, we show that when the myocardium contracts, it applies pressure on the incompressible but mobile cardiac jelly, thus propagating contracting forces to the endocardium. Through live 3D imaging and analysis, we reveal how the endocardium is tethered to the myocardium, causing the endocardium to fold and close the heart lumen when the myocardium contracts. This allows peristaltic pumping of blood and prevents backward flow.

Furthermore, the endocardial folds and tether patterns can be superimposed and are observed at similar locations depending on developmental stages. Our multidisciplinary approach has allowed us to draw a dynamic map of cardiac constituents which will greatly contribute to address numerous fundamental questions in embryonic cardiac development. 